\title{
Corneal densitometry and biomechanical properties in patients with primary congenital glaucoma
}

Laura Morales-Fernández, PhD, ${ }^{\mathrm{a}, \mathrm{b}}$ Blanca Benito-Pascual, MD, ${ }^{\mathrm{a}}$ Pilar Pérez-García, MD, ${ }^{\mathrm{a}}$

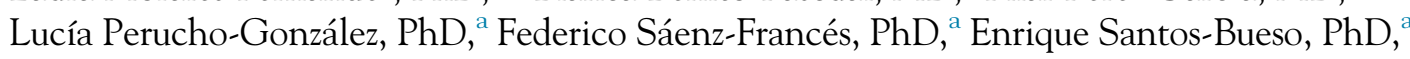
Javier García-Bella, PhD, ${ }^{a}$ Rubén Sánchez-Jean, PhD, ${ }^{a}$ Julián García-Feijoo, PhD, José María Martínez-de-la-Casa, $\mathrm{PhD}^{\mathrm{a}}$

Objective: To describe corneal densitometry, topographic measurements, and biomechanical properties in patients with primary congenital glaucoma (PCG) and healthy patients. To examine correlations between variables and determine their glaucoma diagnostic capacity.

Methods: This was a cross-sectional, case-control study conducted in 50 eyes of 50 patients with PCG (G1) and 40 eyes of 40 healthy patients (G2). The variables determined in each participant were: intraocular pressure, best corrected visual acuity (BCVA), corneal densitometry, topographic data using the Pentacam HR-Scheimpflug imaging system (Oculus Optikgerate $\mathrm{GmbH}$, Wetzlar, Germany), and corneal hysteresis $(\mathrm{CH})$ and corneal resistance factor (CRF) using the Ocular Response Analyzer (Reichert Ophthalmic Instruments, Depew, NY).

Results: Overall densitometry was significatively higher in the PCG group (G1: $17.94 \pm 4.99$ vs G2: $13.25 \pm 1.96, p<$ $0.001)$. $\mathrm{CH}$ (G1: $8.02 \pm 11.35$ vs G2: $11.35 \pm 1.42, p<0.001)$ and CRF (G1: $9.48 \pm 2.83$ vs G2: $10.77 \pm 1.34, p<0.001)$ were lower in the glaucoma group. Mean posterior, central, and anterior elevation and mean keratometry were higher in G1 $p<$ 0.05). In the PCG group, overall densitometry showed significant correlation with $\mathrm{CH}(\mathrm{r}=-0.321, p=0.028)$ and with BCVA $(r=-0.498, p=0.002)$. AUCs (areas under the curve) for $\mathrm{CH}$ and overall densitometry were high ( 0.839 and 0.899 respectively) and the best overall densitometry; $\mathrm{CH}$ and CRF cutoffs were 14.0, 9.3 and 9.2 respectively.

Conclusion: Densitometry is increased, and biomechanical corneal properties are decreased in patients with PCG. Densitometry and visual acuity showed a negative and significant correlation, so this measurement could be used as an indirect parameter of BCVA in the clinical practice.

Primary congenital glaucoma (PCG) is a significant cause of blindness in children. Its diagnosis is based on clinical signs according to a standardized definition agreed upon at the Ninth World Glaucoma Association Consensus meeting by The Childhood Glaucoma Research Network ${ }^{1}$ : an intraocular pressure (IOP) in a child $>21 \mathrm{~mm} \mathrm{Hg}$ accompanied by clinical evidence of glaucoma, such as enlarged corneal diameter, Haab striae, and glaucomatous appearance on the optic nerve head.

Corneal alterations are characteristic of PCG. These may manifest in developing corneas as high IOP (until the age of 3 years) associated with flexible collagen fibers and increased corneal diameter, which produces ruptures in Descemet's membrane (known as Haab striae) because this membrane is less flexible than collagen. ${ }^{2}$ These ruptures connect the aqueous humor with the corneal stroma, leading to edema. ${ }^{3}$ Leukomas and transparency alterations can persist for the life of the patient. The impact of these alterations is huge and results in poor visual acuity and interference with IOP measurements. ${ }^{4}$

Until recently, it was not possible to objectively measure these kinds of corneal abnormalities. However, the advent of concepts such as corneal biomechanics and densitometry on which new machines, such as the Ocular Response Analyzer (ORA, Reichert Ophthalmic Instruments, Depew, NY) and last generation topographers such us the Pentacam HR (Oculus Optikgerate GmbH, Wetzlar, Germany), are based has opened several new possibilities for the diagnosis and management of PCG.

The ORA determines corneal biomechanical properties using an applied force-displacement relationship by an air puff. $^{5}$ Parameters used to characterize the biomechanical properties of the cornea include corneal hysteresis $(\mathrm{CH})$ and corneal resistance factor (CRF). The parameter $\mathrm{CH}$ evaluates the cornea's ability to absorb and dissipate energy, and CRF provides a measure of corneal resistance. Corneal 
Can J Ophthalmol Volume

biomechanical properties were described to be lower in patients with PCG. Indeed, the concept of corneal densitometry is a new and promising approach whereby light scatter is objectively assessed as a measure of abnormalities in corneal transparency. Using the Scheimplug technology of Pentacam devices, measures are expressed in GreyScale Units (GSU; from 0, total transparency, to 100, total opacity). ${ }^{6}$ However, no normative database is included in these devices. ${ }^{5,7}$

In PCG, our group reported a preliminary study, ${ }^{8}$ and densitometric measurements were observed to be higher in patients with PCG. Therefore, we hypothesize that a change in corneal transparency exists in parallel with biomechanical changes induced by PCG. So, measuring the corneal transparency objectively and biomechanical properties may enable us to understand the results of corneal alterations in eyes with PCG.

This study was designed to identify possible differences in corneal densitometry, topographic measurements, and biomechanics between patients with PCG and healthy patients. Variables found to differ, and their correlations were then assessed for their glaucoma diagnostic capacity.

\section{Methods}

For this study, we prospectively recruited 50 patients who had been diagnosed with PCG and were managed at the Department. of Glaucoma of the Hospital Clínico San Carlos, Madrid, Spain and 40 healthy patients. The study protocol was in line with the tenets of the Declaration of Helsinki and was approved by the Ethics Committee of the Hospital Clínico San Carlos, Madrid, Spain. Written informed consent was obtained from each patient and the parents or legal guardian of each child, and each child gave their verbal consent to participate.

Glaucoma participants fulfilled the diagnostic criteria for PCG defined in the last Childhood Glaucoma Research Network consensus meeting ${ }^{1}$ : IOP $>21 \mathrm{~mm} \mathrm{Hg}$ when diagnosed and clinical evidence of glaucoma, including an enlarged corneal diameter, Haab striae, and/or glaucomatous appearance of the optic nerve head. Eyes were also required to have sufficient visual acuity for fixation. When both eyes were eligible, one eye was randomly selected using a randomization list obtained from www.randomization.com. Exclusion criteria were secondary causes of glaucoma (iridocorneal endothelial syndrome, Axenfeld-Rieger syndrome, or Peters anomaly), a systemic disease or situation that could impair cooperation with the tests, corneal surgery, or alterations not related to glaucoma (e.g., corneal transplant or trauma).

From the patients' clinical records, we obtained these data: age at the moment of the study and at diagnosis, IOP measured using Perkins, cup-to-disc ratio, surgeries and treatments until the moment of the study, and the presence of leukoma.
As the control group, 40 healthy volunteers (historic controls) were included. ${ }^{8}$ These healthy volunteers were recruited among those undergoing a routine ophthalmologic exam who agreed to participate in this study. Eyes were defined as normal (in the absence of other features of glaucoma) if they showed a best corrected visual acuity (BCVA) of $\geq 0,8$ (Snellen Scale), an optic nerve cup-disc asymmetry of $\leq 0.2$, and a cup-to-disc ratio of $<0.5$ without focal neuroretinal rim loss, hemorrhage, or pallor. Children were excluded if they had a family history of glaucoma or had been diagnosed with amblyopia or strabismus. All measurements were made in both eyes, but only the data of one randomly selected eye were entered in the analysis.

Firstly, all participants underwent a comprehensive ophthalmologic examination by a single examiner (L.M.F) in which BCVA was measured using Snellen decimal scale. IOP was measured in the glaucoma group using a Goldmann handheld applanation tonometer (Perkins; ClementClarke, Columbus, Ohio). Secondly, all subjects underwent elevation topography using the Pentacam HR and thirdly, ORA measurements were obtained.

These tests were conducted by the same person (R.S.J) with the same device in the automatic testing mode in mesopic conditions and under stable conditions of humidity and temperature. Images were accepted if they were of sufficient quality. Given the good repeatability and reproducibility of the tests, only one test was conducted per participant. ${ }^{6}$ The following topographic and densitometric parameters were recorded using the Pentacam HR: central corneal thickness (CCT); mean $(\mathrm{Km})$ and maximum (Kmax) keratometry; cylinder (Cyl), anterior elevation of the apex; central anterior elevation; maximum anterior elevation; posterior elevation of the apex; central posterior elevation; maximum posterior elevation; and anterior, mid-stromal, and posterior corneal density across its whole area and across the rings $0-2 \mathrm{~mm}, 2-6 \mathrm{~mm}, 6-10 \mathrm{~mm}, 10-12 \mathrm{~mm}$ and overall densitometry. ORA measurements recorded were $\mathrm{CH}$ and $\mathrm{CRF}$.

\section{Statistical Analysis}

Categorical data are provided as their frequency and confidence intervals at $95 \%$ and numerical data as their mean and standard deviation. The Kolmogorov-Smirnov test was used to check the normality of the data. Those showing a non-normal distribution are expressed as their median and interquartile range. Quantitative data were compared using the Students $t$ test and qualitative data using the $\chi 2$ test. Pearson's correlation test was used to detect correlations between the BCVA, ORA, and Pentacam data. The discriminative capacity of a variable for glaucoma was assessed through the area under the receiver operating curve (AUC). All statistical tests were performed using the SPSS package version 18.0 (SPSS Inc, Chicago, Ill.). Significance was set at $p<0.05$. 
Biomechanics and densitometry in primary congenital glaucoma-Morales-Fernández et al.

\section{Results}

The study sample was comprised of 50 eyes with PCG and 40 healthy eyes. The demographic and clinical characteristics of the study participants are provided in Table 1 . Both groups were comparable in terms of age $(p=0.22)$, sex (0.28), and eye examined (0.89). At the time of the study, the two groups were not matched for BCVA and IOP (Table 1).

Clinical characteristics of the PCG group were mean age at the time of the study of was $18.60 \pm 11.65$ years and mean age at diagnosis of PCG was $34.27 \pm 13.60$ months.

\begin{tabular}{|c|c|c|c|}
\hline & Controls $n=40$ & Patients $\mathrm{n}=50$ & $p$ \\
\hline Age (years) & $21.45 \pm 9.94$ & $18.60 \pm 11.65$ & $0.221^{*}$ \\
\hline $\operatorname{Sex}(M / F)$ & $17 / 23$ & $27 / 23$ & $0.282^{\dagger}$ \\
\hline Eye (RE/LE) & $19 / 21$ & $23 / 27$ & $0.892^{+}$ \\
\hline IOPp (mmHg) & $14.07 \pm 2.23$ & $18.18 \pm 3.94$ & $<0.001^{*}$ \\
\hline BCVA & $1.01 \pm 0.30$ & $0.46 \pm 0.34$ & $<0.001^{*}$ \\
\hline \multicolumn{4}{|c|}{$\begin{array}{l}\text { M, male; F, female; RE, right eye; LE, left eye; IOP, intraocular pressure measured } \\
\text { using the Perkins device; BCVA, visual acuity. } \\
{ }^{*} \text { Student } t \text { test } \\
{ }^{\dagger} \chi 2 \text { test. }\end{array}$} \\
\hline
\end{tabular}

BCVA at the time of the study was $0.46 \pm 0.34$ (Snellen Scale), mean cup-to-disc ratio was $0.55 \pm 0.30$, and mean number of hypotensive drops was $1.70 \pm 1.43$. Mean number of glaucoma surgeries were $3.22 \pm 2.88$ per eye. Six eyes had leukoma at the time of the study.

Corneal densitometry values, topographic measurements, and corneal biomechanical properties in both groups are described in Table 2. Figure 1 represents the distribution of the mean densitometric values for each layer (anterior, mid stroma, and posterior cornea) and overall densitometry. Table 3 shows correlation between main variables. Discriminatory capacity of biomechanical corneal properties and overall densitometry are explained in Table 4. Sensitivity and specificity for each measurement are included, and the best $\mathrm{CH}, \mathrm{CRF}$, IOP and overall densitometry cutoffs are also presented in Table 4.

\section{Discussion}

Preservation of visual acuity in patients with PCG is a challenge. Its appropriate management and treatment are crucial, and in most cases, surgery is needed in several

Table 2-Pentacam corneal densitometry and topography measurements. Biomechanical characteristics

\begin{tabular}{|c|c|c|c|c|}
\hline Variable & Controls $n=40$ & Patients $n=50$ & $\mathrm{p}^{*}$ & $95 \% \mathrm{Cl}$ \\
\hline \multicolumn{5}{|c|}{ Anterior densitometry $(120 \mu \mathrm{m})$ in GSU } \\
\hline $0-2 \mathrm{~mm}$ & $16.33 \pm 2.85$ & $25.33 \pm 14.53$ & $<0.001$ & $4.78-13.21$ \\
\hline $2-6 \mathrm{~mm}$ & $15.16 \pm 2.65$ & $23.36 \pm 13.19$ & $<0.001$ & $4.37-12.03$ \\
\hline $6-10 \mathrm{~mm}$ & $14.87 \pm 3.08$ & $21.74 \pm 9.70$ & $<0.001$ & $3.96-4.91$ \\
\hline $10-12 \mathrm{~mm}$ & $23.03 \pm 7.35$ & $25.37 \pm 9.88$ & 0.201 & -1.27 to 5.96 \\
\hline Mean anterior densitometry & $10.82 \pm 2.75$ & $23.46 \pm 10.82$ & $<0.001$ & $3.76-10.13$ \\
\hline \multicolumn{5}{|l|}{ Mid stroma densitometry in GSU } \\
\hline $0-2 \mathrm{~mm}$ & $12.76 \pm 1.48$ & $17.38 \pm 5.34$ & $<0.001$ & $3.04-6.21$ \\
\hline $2-6 \mathrm{~mm}$ & $11.51 \pm 1.27$ & $15.65 \pm 4.00$ & $<0.001$ & $2.94-5.33$ \\
\hline $6-10 \mathrm{~mm}$ & $11.41 \pm 2.04$ & $15.40 \pm 4.70$ & $<0.001$ & $2.52-5.47$ \\
\hline $10-12 \mathrm{~mm}$ & $18.06 \pm 5.58$ & $20.37 \pm 6.81$ & 0.086 & -0.28 to 4.91 \\
\hline Mean mid stroma densitometry & $12.72 \pm 1.77$ & $16.62 \pm 3.64$ & $<0.001$ & $2.73-5.07$ \\
\hline \multicolumn{5}{|c|}{ Posterior densitometry $(60 \mu \mathrm{m})$ in GSU } \\
\hline $0-2 \mathrm{~mm}$ & $10.33 \pm 1.34$ & $14.17 \pm 5.39$ & $<0.001$ & $2.25-5.42$ \\
\hline $2-6 \mathrm{~mm}$ & $9.54 \pm 1.21$ & $13.37 \pm 4.30$ & $<0.001$ & $2.55-5.10$ \\
\hline $6-10 \mathrm{~mm}$ & $9.95 \pm 1.91$ & $13.67 \pm 4.06$ & $<0.001$ & $3.24-6.48$ \\
\hline $10-12 \mathrm{~mm}$ & $14.22 \pm 4.16$ & $16.23 \pm 5.28$ & 0.053 & -0.02 to 4.04 \\
\hline Mean posterior densitometry & $10.54 \pm 1.69$ & $13.98 \pm 3.47$ & $<0.001$ & $2.42-4.64$ \\
\hline \multicolumn{5}{|l|}{ Full thickness densitometry in GSU } \\
\hline $0-2 \mathrm{~mm}$ & $13.13 \pm 1.77$ & $18.95 \pm 6.91$ & $<0.001$ & $3.78-7.85$ \\
\hline $2-6 \mathrm{~mm}$ & $12.08 \pm 1.58$ & $17.45 \pm 5.91$ & $<0.001$ & $3.63-7.12$ \\
\hline $6-10 \mathrm{~mm}$ & $12.07 \pm 2.22$ & $16.93 \pm 5.18$ & $<0.001$ & $3.24-6.48$ \\
\hline $10-12 \mathrm{~mm}$ & $18.44 \pm 5.44$ & $20.66 \pm 6.71$ & 0.093 & -0.32 to 4.77 \\
\hline Overall densitometry & $13.25 \pm 1.96$ & $17.94 \pm 4.99$ & $<0.001$ & $3.27-6.34$ \\
\hline \multicolumn{5}{|l|}{ Topographic measurements } \\
\hline AAE & $2.33 \pm 2.30$ & $3.44 \pm 3.97$ & 0.100 & -0.22 to 2.45 \\
\hline ACE & $2.40 \pm 2.34$ & $3.57 \pm 6.53$ & 0.284 & -0.83 to 3.18 \\
\hline AME & $3.08 \pm 2.58$ & $8.28 \pm 8.77$ & $<0.001$ & $2.59-7.82$ \\
\hline PAE & $1.03 \pm 3.20$ & $4.20 \pm 8.57$ & 0.018 & $0.55-5.80$ \\
\hline PCE & $1.35 \pm 3.38$ & $5.53 \pm 13.59$ & 0.043 & $0.15-8.22$ \\
\hline PME & $8.90 \pm 9.57$ & $9.42 \pm 6.02$ & $<0.001$ & $12.57-28.47$ \\
\hline KM & $43.57 \pm 1.8$ & $41.61 \pm 3.06$ & $<0.001$ & -2.99 to -0.91 \\
\hline $\mathrm{K} 2$ & $43.97 \pm 1.93$ & $40.70 \pm 3.23$ & $<0.001$ & -4.36 to -2.18 \\
\hline $\mathrm{CCT}$ & $559.63 \pm 29.95$ & $559.18 \pm 50.15$ & 0.961 & -17.41 to 16.52 \\
\hline \multicolumn{5}{|l|}{ Biomechanical variables } \\
\hline $\mathrm{CH}$ & $11.35 \pm 1.42$ & $8.02 \pm 11.35$ & $<0.001$ & -4.14 to -2.51 \\
\hline CRF & $10.77 \pm 1.34$ & $9.48 \pm 2.83$ & $<0.001$ & -2.22 to -0.35 \\
\hline \multicolumn{5}{|c|}{$\begin{array}{l}\text { GSU, grey scale units; AAE, anterior apex elevation; ACE, anterior central elevation; AME, anterior maximum elevation; PAE, posterior apex elevation; PCE, posterior central } \\
\text { elevation; PME, posterior maximum elevation; KM, mean keratometry; K2, keratometry of steepest meridian; CCT, central corneal thickness (microns); CH, corneal hystere- } \\
\text { sis; CRF, corneal resistance factor; Cl, confidence interval. } \\
{ }^{*} \text { Difference significant (Student's } t p<0.05 \text { ). }\end{array}$} \\
\hline
\end{tabular}


Can J Ophthalmol Volume $\mathbf{\square}$, Number $\mathbf{\square}, \mathbf{\square} 2021$

1.a.

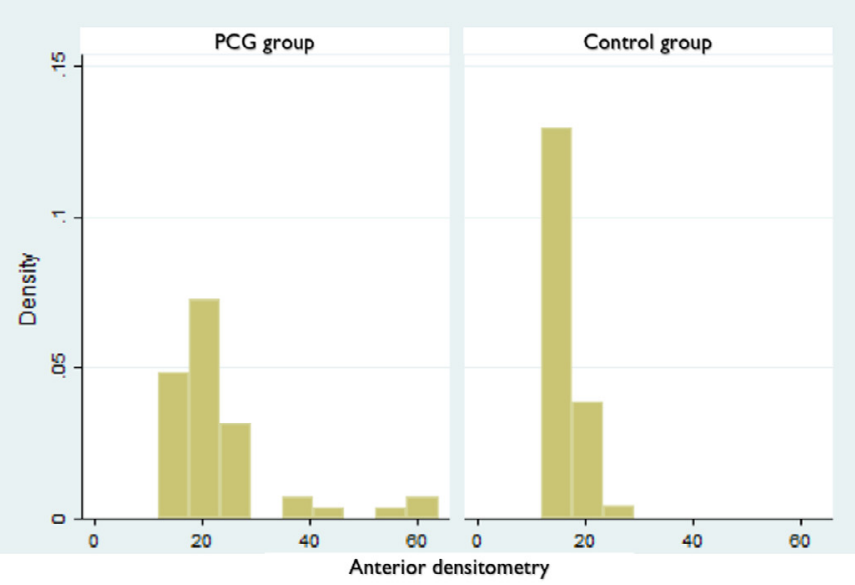

1.b.

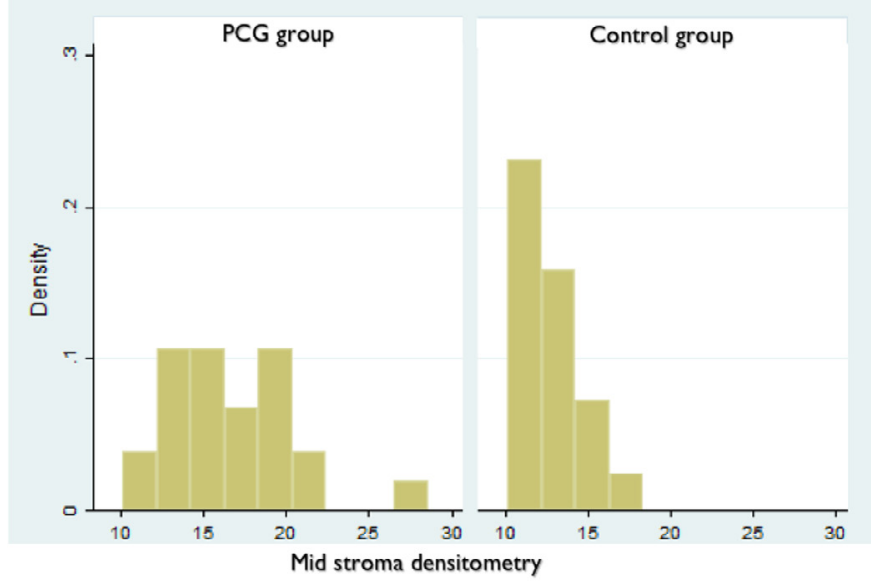

1.c.

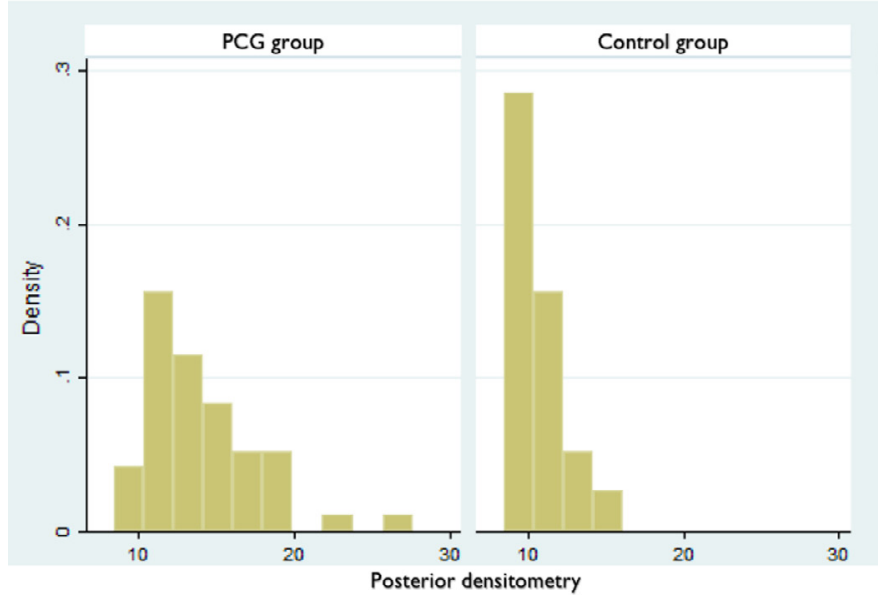

Figure 1-These figures represent the distribution of densitometric values obtained using Pentacam in both groups: (1.a. Anterior densitometry; 1.b. Mid stroma densitometry; 1.c. posterior densitometry; 1.d. Overall densitometry). PCG: primary congenital glaucoma $X$ axis (densitometry values): units expressed as Grey scale units (GSU).

occasions. However, despite adequate management, the presence of complications such as cataract, corneal opacity, amblyopia, or terminal visual field loss in these young patients is not uncommon. ${ }^{9}$ Although the diagnosis of PCG is essentially clinical, by using new topographers and/or tonometers (ORA) clinicians can obtain relevant data to complement the examination of these patients in routine clinical practice.

In the present study, all the measured values of densitometry were significantly higher in the glaucoma group, for the corneal $0-2 \mathrm{~mm}, 2-6 \mathrm{~mm}, 6-10 \mathrm{~mm}$ annuli and across the whole cornea. Excepting 10-12 $\mathrm{mm}$ annuli 
Biomechanics and densitometry in primary congenital glaucoma-Morales-Fernández et al.

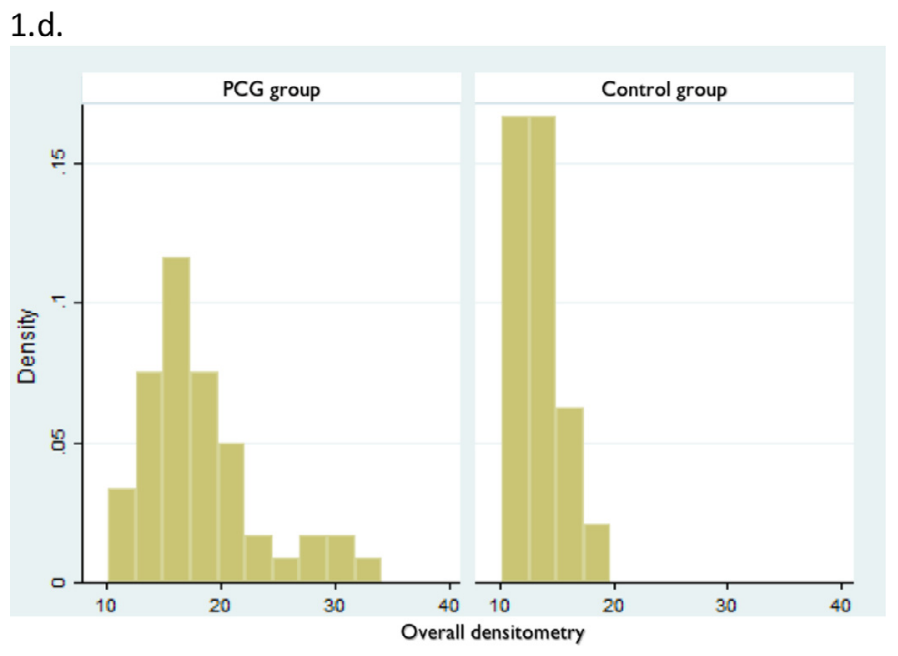

Figure 1-Continued

Table 3-Pearson's correlation coefficients between variables

\begin{tabular}{llrr}
\hline & Group & Pearson's correlation & $P$ value \\
\hline BCVA-DensT & Patients & -0.498 & 0.002 \\
CH-DensT & Controls & 0.117 & 0.497 \\
& Patients & -0.321 & 0.028 \\
CCT-CH & Controls & 0.589 & $<0.001$ \\
& Patients & 0.220 & 0.143 \\
CCT-DensT & Controls & -0.270 & 0.090 \\
& Patients & -0.122 & 0.513 \\
IOP-DensT & Controls & -0.302 & 0.105 \\
& Patients & 0.265 & 0.174
\end{tabular}

BCVA, visual acuity; DensT, overall densitometry; IOP, intraocular pressure in $\mathrm{mmHg}$ (Perkins); $\mathrm{CH}$, corneal hysteresis; CRF, corneal resistance factor; CCT, central corneal thickness.

measurements, full-thickness densitometry values ranged from 12.07 GSU to $13.13 \mathrm{GSU}$ in the healthy group and ranged from 16.93 GSU to 18.95 GSU in the PCG group.

First, no differences were found between both groups in $10-12 \mathrm{~mm}$ annuli densitometric values. In pseudoexfoliation (PEX) syndrome, similar values were reported from 25.21 to 31.17 GSU in external $10-12 \mathrm{~mm}$ zone, much higher than inner values. These values near to the limbus has been reported always higher than inner values. This can be owing to the lack of transparency in this area and transient scleral-corneal tissues.

Second, considering the different corneal layers, higher densitometric measurements were found in the anterior layer in both groups compared with mid and posterior zone measurements. The highest densitometric values were also found in the anterior layer in PCG group (from 21.74 to $25.33 \mathrm{GSU})$. Tekin et al. ${ }^{10}$ reported a correlation between endothelial abnormalities and corneal densitometry and argued that corneal densitometry could play an important role as an indicator of healthy endothelium. This could be very interesting in PCG patients. ${ }^{11,12}$ Typical Haab striae in PCG are located in deep cornea (endothelial ruptures); however, these measurements suggest that superficial corneal layers are also involved in corneal densitometry. Corneal changes were assessed previously using confocal microscopy in patients with PCG. ${ }^{13}$ Interestingly, although no differences were reported between the number of keratocytes in buphthalmic eyes and healthy eyes in the anterior stroma or in the posterior stroma, the cell density of basal epithelial cells was reported to be significantly higher in buphthalmic eyes relative to healthy eyes. All these changes could be involved in increasing densitometric values.

Similar alterations in anterior densitometry were reported in PEX syndrome. ${ }^{14}$ In accordance with our results, anterior density was higher than mid and posterior density, and it is remarkable that high range of values was reported (from 17.9 to 49.7). In PEX syndrome, material deposits exist in the anterior stroma of the corneas, and these changes could be responsible for the increased anterior density measurements. ${ }^{14}$

Third, densitometric values in our study were higher in inner annuli (18.95 GSU) than outer annuli (from 16.93 to 17.45 GSU) in both groups. This could explain inverse

Table 4-Diagnostic capacity for each parameter (area under ROC curve)

\begin{tabular}{|c|c|c|c|c|c|c|c|}
\hline & AUC & SD & S $80 \%$ & S 90\% & Best cutoff & S (cutoff) & E (cutoff) \\
\hline DensT & 0.839 & 0.041 & 76.00 & 58.00 & $>14.0$ & 84.00 & 75.00 \\
\hline $\mathrm{CH}$ & 0.899 & 0.359 & 80.85 & 76.60 & $<9.3$ & 76.60 & 97.22 \\
\hline IOP & 0.827 & 0.059 & 75.00 & 71.43 & $>16.0$ & 71.43 & 93.33 \\
\hline CRF & 0.676 & 0.061 & 55.32 & 55.32 & $<9.2$ & 55.32 & 94.44 \\
\hline
\end{tabular}

DensT, overall densitometry; IOP, intraocular pressure in mmHg (Perkins); CH, corneal hysteresis; CRF, corneal resistance factor; AUC, area under the ROC curve; SD, standard deviation; S, sensibility; E, specificity. 


\section{Can J Ophthalmol Volume}

, Number $\mathbf{\square} 2021$

correlation with BCVA reported in this study. In PEX syndrome ${ }^{14}$ an increased densitometry was also reported in the central layer of the cornea (ranged from 12.8 to $29.1 \mathrm{GSU}$ ). Abnormalities of stromal keratocytes and altered stromal hydration because of functional impairment in the endothelium may be the cause of this central increasement in both types of glaucoma.

We also detected significant differences between cases and controls in topographic variables, in posterior apex, maximum, and central elevation $(1.03 \pm 3.20$ vs $4.20 \pm$ $8.57, p=0.018 ; 8.90 \pm 9.57$ vs $9.42 \pm 6.02, p<0.001 ; 1.35$ \pm 3.38 vs $5.53 \pm 13.59, p<0.001$ respectively), and mean and steepest meridian keratometry $(43.57 \pm 1.80$ vs 41.61 $\pm 3.06, p<0.001 ; 43.97 \pm 1.93$ vs $40.70 \pm 3.23, p<$ $0.001)$. This could be owing to corneal deformations in posterior elevation and curvature owing to the high IOP in flexible developing corneas. ${ }^{6}$ Previous studies described similar results. ${ }^{7}$

In the present study, we observed lower $\mathrm{CH}$ and $\mathrm{CRF}$ in PCG patients than controls (CH: $11.35 \pm 1.42$ vs $8.02 \pm$ $11.35, p<0.001$; CRF: $11.77 \pm 1.34$ vs $9.48 \pm 2.83, p<$ 0.001 ). Studies addressing the issue of how $\mathrm{CH}$ varies in different disease states have detected a decrease in $\mathrm{CH}$ and CRF in conditions in which collagen is affected (keratoconus, Fuchs dystrophy) and in patients with ocular hypertension (with or without glaucoma), and a relationship has also been noted with modifications to the lamina cribosa. ${ }^{15}$ Perucho et al. ${ }^{7}$ described similar values in biomechanical corneal properties in patients with PCG $(\mathrm{CH}$ was 8.55 in PCG vs 11.19 in healthy eyes).

Confirming our hypothesis, inverse correlation between overall densitometry and $\mathrm{CH}$ in the PCG group was found. There is some evidence for corneal biomechanical alterations together with stromal changes in PEX. $\mathrm{CH}$, endothelial cell density, and corneal nerve densities were found decreased in PEX eyes and PEX glaucoma eyes. ${ }^{16,17}$

In contrast, other authors published no significant correlation between stromal changes using confocal microscopy (the keratocyte density in anterior and/or posterior stroma) and $\mathrm{CH}$ or CRF in PCG patients $(\mathrm{r}=0.29) .{ }^{18}$

No correlation was found between CCT and CH or CCT and densitometry. CCT was considered the main interference factor with IOP measurements using Goldmann tonometers; however, this lack of correlation between CCT and $\mathrm{CH}$ or densitometry could explain why some studies consider $\mathrm{CH}$ and CCT as an independent factor to interfere over IOP Goldmann measurements. ${ }^{19}$ Despite this, more studies are necessary to determine the influence of densitometry over IOP Goldmann measurements. ${ }^{4}$

In this study we confirm a significant correlation between BCVA and overall densitometry in the PCG group ( $\mathrm{r}$ $=-0.498)$. We reported similar results in our preliminary study. ${ }^{8}$ Both studies highlight that this relationship could be an objective way to measure how corneal alterations can affect BCVA independently of other causes (glaucoma optic neuropathy, cataract, vitreo-retinal changes, etc,). ${ }^{20}$ In routine clinical practice, this tool could be useful to monitor corneal transparency and its impact on visual acuity. However, it should be noted that there is no normative database either in the Pentacam HR software or ORA, so this study offers normality data for healthy children and for patients with PCG. In this study, best cutoffs are detailed for overall densitometry (>14.0) and for $\mathrm{CH}(<9.3)$. This information is essential for patient follow-up in the clinical practice.

The diagnostic capacity of corneal densitometry was found to be high, with an AUC of 0.839 , and higher than the value of 0.827 obtained for IOP measured with the Perkins device. Moreover, the diagnostic capacity of corneal biomechanics was also fairly good, with an AUC of 0.899 obtained for $\mathrm{CH}$. Despite a clinical diagnosis of PCG, our study shows that biomechanics and densitometry values differ between patients with PCG and healthy patients and that densitometry measurements have a good discriminatory capacity.

The limitations of our study include the scant number of patients, its cross-sectional design, and its selection bias because only patients with good fixation were selected (determining better visual acuity, lower severity of glaucoma, and improved corneal transparency).

In conclusion, our findings reveal that those patients with PCG feature higher corneal densitometry and lower corneal biomechanical properties than healthy patients. In the patients with PCG, negative correlation was observed between overall densitometry and $\mathrm{CH}$ and between BCVA and overall corneal densitometry, which could be useful to assess the impact of corneal alterations on BCVA in the clinical practice. New devices, such as Pentacam HR or ORA, are useful tools and offer additional information to describe corneal alterations in this disease.

\section{References}

1. Beck A, Chang TC, Freedman S, et al. Section 1: Definition, classification, differential diagnosis. In: Weinreb RN, Grajewski A, Papadopoulos M, Grigg J, Freedman S, editors. World Glaucoma Association Consensus Series-9: Childhood Glaucoma. Amsterdam, The Netherlands: Kugler Publications; 2013. p. 3-10.

2. Morin JD, Coughlin WR. Corneal changes in primary congenital glaucoma. Trans Am Ophthalmol Soc 1980;78:12331.

3. Benito-Pascual B, Pascual-Prieto J, Martínez-de-la-Casa JM, Sáenz-Francés F, Santos-Bueso E. Haab striae: Optical coherence tomographic analysis. J Fr Ophtalmol 2019;42(1):11-5.

4. Kaushik S, Pandav SS, Banger A, Aggarwal K, Gupta A. Relationship between corneal biomechanical properties, central corneal thickness, and intraocular pressure across the spectrum of glaucoma. Am J Ophthalmol 2012;153:840-9.

5. Perucho-González L, Martínez de la Casa JM, MoralesFernández L, Bañeros-Rojas P, Saenz-Francés F, García-Feijoó J. Intraocular pressure and biomechanical corneal properties measure by ocular response analyser in patients with primary congenital glaucoma. Acta Ophthalmol 2016;94:e293-7. 
Biomechanics and densitometry in primary congenital glaucoma-Morales-Fernández et al.

6. Ní Dhubhghaill S, Rozema JJ, Jongenelen S, Ruiz Hidalgo I, Zakaria N, Tassignon MJ. Normative values for corneal densitometry analysis by Scheimpflug optical assessment. Invest Ophthalmol Vis Sci 2014;55:162-8.

7. Perucho-González L, Sáenz-Frances F, Morales-Fernandez L, Martinez-de-la-Casa JM, Méndez-Hernández CD, SantosBueso E, Brookes JL, García-Feijoó J. Structural and biomechanical corneal differences between patients suffering from primary congenital glaucoma and healthy volunteers. Acta Ophthalmol 2017;95:e107-e12.

9. Morales-Fernández L, Perucho-González L, Martinez-de-laCasa JM, et al. Corneal densitometry and topography in patients with primary congenital glaucoma. J Fr Ophtalmol 2020;43:697-703.

8. Thiagalingam S, Jakobiec FA, Chen T, Michaud N, Colby KA, Walton DS. Corneal anomalies in newborn primary congenital glaucoma. J Pediatr Ophthalmol Strabismus 2009; 46:241-4.

10. Tekin K, Sekeroglu MA, Kiziltoprak H, Yilmazbas P. Corneal densitometry in healthy corneas and its correlation with endothelial morphometry. Cornea 2017;36:1336-42.

11. Patil B, Tandon R, Sharma N, Verma M, Upadhyay AD, Gupta V, Sihota R. Corneal changes in childhood glaucoma. Ophthalmology 2015;122:87-92.

12. Pekel G, Özbakış F, Bahar A, Pekel E, Çetin EN. Correlations of corneal optical densitometry, endothelial hexagonality percentage, and epithelium thickness. Curr Eye Res 2018;43:170-4.

13. Mahelková G, Filous A, Odehnal M, Cendelín J. Corneal changes assessed using confocal microscopy in patients with unilateral buphthalmos. Invest Ophthalmol Vis Sci 2013;54:4048-53.

14. Zheng X, Shiraishi A, Okuma S, et al. In vivo confocal microscopic evidence of keratopathy in patients with pseudoexfoliation syndrome. Invest Ophthalmol Vis Sci 2011;52:1755-61.
15. Gatzioufas Z, Labiris G, Stachs O, et al. Biomechanical profile of the cornea in primary congenital glaucoma. Acta Ophthalmol 2013;91:e29-34.

16. Cankaya AB, Anayol A, Özcelik D, et al. Ocular response analyzer to assess corneal biomechanical properties in exfoliation syndrome and exfoliative glaucoma. Graefes Arch Clin Exp Ophthalmol 2012;250:255-60.

17. Yazgan S, Celik U, Alagöz N, et al. Corneal biomechanical comparison of pseudoexfoliation syndrome, pseudoexfoliative glaucoma and healthy subjects. Curr Eye Res 2015;40:470-5.

18. Gatzioufas Z, Labiris G, Stachs O, et al. Biomechanical profile of the cornea in primary congenital glaucoma. Acta Ophthalmol 2013;91:e29-34.

19. Garzón N, Poyales F, Illarramendi I, et al. Corneal densitometry and its correlation with age, pachymetry, corneal curvature, and refraction. Int Ophthalmol 2017;37:1263-8.

20. Urbaniak D, Seredyka-Burduk M, Błoch W, Malukiewicz G, Kałużny BJ. Scheimpflug camera measurement of optical density of the corneal epithelium, stroma, and endothelium in patients with pseudoexfoliation syndrome. Med Sci Monit 2018;24:5826-31.

\section{Footnotes and Disclosure}

The authors have no proprietary or commercial interest in any materials discussed in this article.

From the aphthalmology Unit, Hospital Clinico San Carlos, Instituto de Investigacion Sanitaria del Hospital Clinico

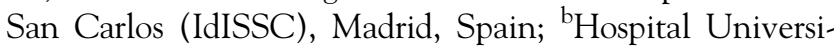
tario Quirónsalud Madrid, Madrid, Spain.

Correspondence to: Laura Morales-Fernandez, Profesor Martin Lagos Street, Madrid, Spain. lauramoralesfernandez@gmail. com 